\title{
Regular and chaotic flow behavior and orientational dynamics of tumbling nematics
}

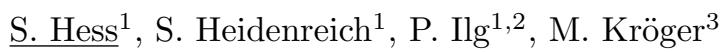 \\ ${ }^{1}$ Institut f. Theoretische Physik, Technische Universität Berlin, Hardenbergstraße 36 D-10623 Berlin, Germany \\ ${ }^{2}$ Départment de Physique des Matériaux, UCB Lyon1, F-69622 Villeurbanne, France \\ ${ }^{3}$ Polymer Physics, ETH Zürich, Wolfgang-Pauli-Straße 10, HCI-537, CH-8093 Zürich, Switzerland
}

The flow behavior of (both low molecular weight and polymeric) liquid crystals is strongly affected by the coupling between the flow and the molecular orientation. Nematic liquid crystals which respond with a time-dependent orientational behavior to an applied steady shear flow rather than a stationary flow alignment are called 'tumbling nematics'. The time-dependent phenomena can be rather complex. Different types of periodic behavior referred to as (ordinary) tumbling, wagging, kayaking tumbling and kayaking wagging have been idendified in experiments and in theoretical descriptions [1].

A relatively simple model based on a nonlinear equation for the second rank alignment tensor which can be derived form irreversible thermodynamics [2, $3,4]$ or from a generalized Fokker-Planck equation $[5,6]$ revealed a more complex and even chaotic behavior for certain model parameters and specific values of the applied shear rate [7]. Chaotic behavior was also found from a solution of a Fokker-Planck equation for the orientational distribution function involving 65 components rather than the 5 independent components of the second rank alignment tensor [8]. Further theoretical studies on the periodic and chaotic orientational and rheological behavior were presented in [9] and in [10].

Here the following further points are addressed:

i) How sensitive are the solutions of the model equation against time-dependent distortions of the applied shear rate since fluctuating contributions to the shear rate do occur in experiments. It is demonstrated that periodic and chaotic solutions can be surprisingly robust against such distortions.

ii) We amend the previous theory based on a Landau de Gennes free energy which includes terms up to $4^{\text {th }}$ order in the alignment tensor and which does not impose a bound on the magnitude of the alignment tensor by a version which includes arbitrary high orders and does impose a realistic bound. This point is of importance for numerical solutions, in particular in spatially inhomogeneous situations where run-away solutions might lead to unphysically large values of the alignment.
The qualitative dynamic behavior obtained previously is hardly changed under the mentioned modifications of the model equations. Of course, there are (small) quantitative changes of the parameter ranges where the various types of the orientational behavior is found.

iii) Some results are shown for a spatially inhomogeneous system.

\section{References}

[1] R.G. Larson, The Structure and Rheology of Complex Fluids, Oxford University Press, Oxford, UK (1999).

[2] S. Hess, Z. Naturforsch. 30a, 728 (1975), 31a 1507 (1976).

[3] P. D. Olmsted and P. Goldbart, Phys. Rev. A 41, 4578 (1990); Phys. Rev. A 46, 4966 (1992).

[4] C. Pereira Borgmeyer and S. Hess, J. NonEquilib. Thermodyn. 20, 359 (1995).

[5] S. Hess, Z. Naturforsch. 31a, 1034 (1976).

[6] M. Doi, Ferroelectrics 30, 247 J. Polym. Sci. Polym. Phys. 19, 229 (1981)

[7] G. Rienäcker, M. Kröger, and S. Hess, Phys. Rev. E 66, 040702(R) (2002); Physica A 315, 537 (2002).

[8] M. Grosso, R. Keunings. S. Crescitelli, and P.L. Maffettone, Phys. Rev. Lett. 86, 3184 (2001).

[9] M. G. Forest, Q. Wang, and R. Zhou, Rheol. Acta 86, 80 (2004) S. Fielding and P. Olmsted, Phys. Rev. Lett. 92, 084502 (2004); B. Chakrabarti, M. Das, C. Dasgupta, S. Ramaswamy, and A.K. Sood, Phys. Rev. Lett. 92, 055501 (2004) .

[10] S. Hess and M. Kröger, J. Phys.: Condens. Matter 163835 (2004); in: Computer simulations bridging liquid crystals and polymers, Eds. P. Pasini, C. Zannoni, S. Zumer (Kluwer Dordrecht), (2005) 295-334 